\title{
Retraction Note to: A randomized phase II study of everolimus for advanced pancreatic neuroendocrine tumors in Chinese patients
}

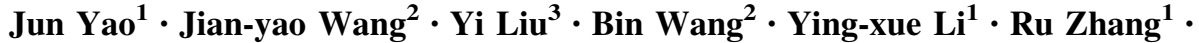 \\ Li-Sheng Wang ${ }^{1} \cdot$ Lei Liu $^{2}$
}

Published online: 21 July 2015

(c) Springer Science+Business Media New York 2015

\section{Retraction Note to: Med Oncol (2014) 31:251 \\ DOI 10.1007/s12032-014-0251-x}

This article has been retracted by the authors because the authors discovered statistical errors which need further validation.

The online version of the original article can be found under doi:10.1007/s12032-014-0251-x.

Li-Sheng Wang

wangls168@163.com

$\triangle$ Lei Liu

liulei3322@yahoo.com.cn

1 Department of Gastroenterology, Shenzhen Municipal People's Hospital, Jinan University of Medical Sciences, 1017 East Gate Road, Shenzhen 518020,

Guangdong Province, China

2 Department of General Surgery, Shenzhen Children's Hospital, Shenzhen 518026, Guangdong Province, China

3 Translational Medicine Center, Laboratory of Oncology, Affiliated Hospital of Academy of Military Medical Sciences, Beijing 100071, China 